\title{
A GENERALIZED POINCARÉ-LELONG FORMULA
}

\author{
MATS ANDERSSON*
}

\begin{abstract}
We prove a generalization of the classical Poincaré-Lelong formula. Given a holomorphic section $f$, with zero set $Z$, of a Hermitian vector bundle $E \rightarrow X$, let $S$ be the line bundle over $X \backslash Z$ spanned by $f$ and let $Q=E / S$. Then the Chern form $c\left(D_{Q}\right)$ is locally integrable and closed in $X$ and there is a current $W$ such that $d d^{c} W=c\left(D_{E}\right)-c\left(D_{Q}\right)-M$, where $M$ is a current with support on $Z$. In particular, the top Bott-Chern class is represented by a current with support on $Z$. We discuss positivity of these currents, and we also reveal a close relation with principal value and residue currents of Cauchy-Fantappiè-Leray type.
\end{abstract}

\section{Introduction}

Let $f$ be a holomorphic (or meromorphic) section of a Hermitian line bundle $L \rightarrow X$, and let $[Z]$ be the current of integration over the divisor $Z$ defined by $f$. The Poincaré-Lelong formula states that

$$
d d^{c} \log (1 /|f|)=c_{1}\left(D_{L}\right)-[Z],
$$

where $c_{1}\left(D_{L}\right)$ is the first Chern form associated with the Chern connection $D_{L}$ on $L$, i.e., $c_{1}\left(D_{L}\right)=\aleph \Theta_{L}$, where $\Theta_{L}$ is the curvature; here and throughout this paper $\aleph=i / 2 \pi$ and $d^{c}=\kappa(\bar{\partial}-\partial)$ so that

$$
d d^{c}=\frac{i}{\pi} \partial \bar{\partial}=2 \aleph \partial \bar{\partial} .
$$

If $U$ is the meromorphic section of the dual bundle $L^{*}$ such that $U \cdot f=1$, then $R=\bar{\partial} U$ is a $(0,1)$-current, and we have the global factorization

$$
[Z]=R \cdot D_{L} f / 2 \pi i .
$$

If $A=-2 \aleph \partial \log (1 /|f|)$, then clearly $d A=\bar{\partial} A=c_{1}\left(D_{L}\right)-[Z]$, and it is easily checked that $A=U \cdot D_{L} f / 2 \pi i$. In this paper we consider analogous formulas for a holomorphic section $f$ of a higher rank bundle, and our main result is the following generalization of the Poincaré-Lelong formula.

\footnotetext{
* The author was partially supported by the Swedish Natural Science Research Council. Received February 15, 2006.
} 
THEOREM 1.1. Let $f$ be a holomorphic section of the Hermitian vector bundle $E \rightarrow X$ of rank $m$. Let $Z=\{f=0\}$, let $S$ denote the (trivial) line bundle over $X \backslash Z$ generated by $f$, and let $Q=E / S$, equipped with the induced Hermitian metric.

(i) The Chern form c $\left(D_{Q}\right)$ is locally integrable in $X$ and its natural extension to $X$ is closed. Moreover, the forms $\log |f| c\left(D_{Q}\right)$ and

$$
|f|^{2 \lambda} \frac{\aleph \partial|f|^{2} \wedge \bar{\partial}|f|^{2}}{|f|^{4}} \wedge c\left(D_{Q}\right), \quad \lambda>0
$$

are locally integrable in $X$, and

$$
M=\lim _{\lambda \rightarrow 0^{+}} \lambda|f|^{2 \lambda} \frac{\kappa \partial|f|^{2} \wedge \bar{\partial}|f|^{2}}{|f|^{4}} \wedge c\left(D_{Q}\right)=d d^{c}\left(\log |f| c\left(D_{Q}\right)\right) \mathbf{1}_{Z}
$$

is a closed current of order zero with support on $Z$. If $\operatorname{codim} Z=p$, then

$$
M=M_{p}+M_{p+1}+\cdots+M_{\min (m, n)},
$$

where $M_{k}$ has bidegree $(k, k)$, and

$$
M_{p}=\sum \alpha_{j}\left[Z_{j}^{p}\right]
$$

where $Z_{j}^{p}$ are the irreducible components of codimension precisely $p$, and $\alpha_{j}$ are the Hilbert-Samuel multiplicities of $f$.

(ii) There is a current $W$ of bidegree $(*, *)$ and order zero in $X$ which is smooth in $X \backslash Z$, and with logarithmic singularity at $Z$, such that

$$
d d^{c} W=c\left(D_{E}\right)-C\left(D_{Q}\right)-M,
$$

where $C\left(D_{Q}\right)$ denote the natural extension of $c\left(D_{Q}\right)$.

Here $c(D)$ denotes the Chern form with respect to the Chern connection $D$ associated to the Hermitian structure, i.e., $c(D)=\operatorname{det}(\kappa \Theta+I)$, where $\Theta=D^{2}$ is the curvature tensor. We let $c_{k}(D)$ denote the component of bidegree $(k, k)$.

For an explicit expression for $W$, see Definition 4.4 in Section 4. If $W_{k}$ denotes the component of bidegree $(k, k)$, then (1.4) means that

$$
d d^{c} W_{k-1}=c_{k}\left(D_{E}\right)-c_{k}\left(D_{Q}\right)-M_{k}
$$

Since $Q$ has rank $m-1, c_{m}\left(D_{Q}\right)=0$, and therefore

$$
d d^{c} W_{m-1}=c_{m}\left(D_{E}\right)-M_{m},
$$


which means that the current $M_{m}$ represents the top degree Bott-Chern class $\hat{c}_{m}(E)$. It also follows that the Bott-Chern class $\hat{c}_{k}(E)$ is equal to $\hat{c}_{k}(Q)$ if $k<p$.

If $E$ is a line bundle, then, see Definition $4.4, W=W_{0}=\log (1 /|f|)$, so (1.5) is the then usual Poincaré-Lelong formula.

In [8] Bott and Chern developed a method of transgression which in particular gives a form $w$ in $X \backslash Z$ such that $d d^{c} w=c\left(D_{E}\right)-c\left(D_{Q}\right)$. It is not unexpected that one can extend this construction across $Z$ by a careful analysis of the occurring singularities at $Z$. In the recent paper [17], Meo proves (1.5) for $k=p$. Previously this formula was proved in [7] in the case when $f$ defines a complete intersection, i.e., $p=m$. A variety of analogous formulas for $d$ rather than $d d^{c}$ are constructed in quite general (non-holomorphic) situations in [12], [13], [14], and [15].

Clearly $M_{p}$ is always a positive current. It follows from (1.3) that $M_{k}$ is positive if $c_{k-1}\left(D_{Q}\right)$ is a positive form. For an even more precise formula for $M$, see Proposition 7.5.

Let us say that $E$ is positive if $E^{*}$ is Nakano negative.

THEOREm 1.2. Assume that $E$ is positive. Then $c\left(D_{E}\right)$ is a positive form, $C\left(D_{Q}\right)$ and $M$ are positive currents, and (one can choose $W$ such that) $W$ is positive where $|f| \leq 1$.

If $A=-2 \ltimes \partial W$ we have, cf., (1.4),

$$
\bar{\partial} A=d A=c\left(D_{E}\right)-c\left(D_{Q}\right)-M .
$$

In [1] we introduced a residue current $R=R_{p}+\cdots+R_{\min (m, n)}$, associated with $f$, with support on $Z$, where $R_{k}$ is a $(0, k)$-current with values in $\Lambda^{k} E^{*}$, and a principal value current $U=U_{1}+\cdots+U_{m}$ such that $\left(\delta_{f}-\bar{\partial}\right) U=1-R$, where $\delta_{f}$ denotes contraction with $f$. When $E$ is a line bundle, then $U=1 / f$ and $R=\bar{\partial}(1 / f)$. In analogy to (1.1) we can factorize $M_{p}$ as

$$
M_{p}=R_{p} \cdot\left(D_{E} f\right)^{p} / p !
$$

this was proved in [2]. We have a similar, but somewhat more involved, formula for the whole current $M$, see (6.4) in Section 6. In a similar way we can express $A$ and $c\left(D_{Q}\right)$, see (6.5) and (6.6), in terms of the current $U$.

REMARK 1. Let $f_{1}, \ldots, f_{r}$ be holomorphic sections of $E$ and let $Z$ be the analytic set where they are linearly dependent. Moreover, let $S$ be the trivial rank $r$-subbundle of $E$ over $X \backslash Z$ generated by $f_{j}$ and let $Q=E / S$. Then $c\left(D_{Q}\right)$ has a natural current extension $C\left(D_{Q}\right)$ across $Z$ and there is a closed current $M$ of bidegree $(*, *)$ with support on $Z$ and a current $A$ such that

$$
d A=\bar{\partial} A=c\left(D_{E}\right)-C\left(D_{Q}\right)-M .
$$


This can be proved by a small modification of the argument in this paper; in the case $Z$ has generic dimension such a formula was proved already in [14], and the general case should be contained in [15]. It follows from (1.7) that the current $M_{k}$ is a representative for $c_{k}\left(D_{E}\right)$ for $k>m-r$.

However, we have no analogous formula for $d d^{c}$.

As indicated above, the proof of Theorem 1.1 relies on the construction in [8], combined with a careful control of the singularities at $Z$. To begin with one constructs a form $v$ in $X \backslash Z$ such that

$$
d d^{c} v=c\left(D_{E}\right)-c\left(D_{S}\right) c\left(D_{Q}\right) .
$$

By Hironaka's theorem and toric resolutions, following [4] and [18], we can prove that this equality has meaning in the current sense across $Z$. Here a crucial point is an explicit formula for the Chern form $c\left(D_{Q}\right)$ (Proposition 4.2) from which it is easy to conclude that $c\left(D_{Q}\right)$ has a smooth extension across the singularity after an appropriate blow-up. By the usual Poincaré-Lelong formula, $c\left(D_{S}\right)-1=d d^{c} \log (1 /|f|)$ outside $Z$, and we can conclude that (1.4) holds (if the capitals denote the natural extensions across $Z$ ) with

$$
W=\log (1 /|f|) C\left(D_{Q}\right)-V,
$$

and $M=d d^{c}\left(\log |f| C\left(D_{Q}\right)\right) \mathbf{1}_{Z}$. Theorem 1.2 follows essentially by applying ideas in [8].

In Section 7 we discuss the positivity and prove Theorem 1.2, essentially by applying ideas from [8]. The paper is concluded by some examples.

\section{Preliminaries}

We first recall the differential geometric definition of Chern classes. Let $E \rightarrow$ $X$ be any differentiable complex vector bundle over a differential manifold $X$, with connection $D: \mathscr{E}_{k}(X, E) \rightarrow \mathscr{E}_{k+1}(X, E)$ and curvature tensor $D^{2}=\Theta \in$ $\mathscr{E}_{2}(X$, End $E)$. The connection $D=D_{E}$ induces in a natural way a connection $D_{\text {End } E}$ on the bundle End $E$ by the formula $D g \cdot \xi=D(g \cdot \xi)-g \cdot D \xi$, and in a similar way there is a natural connection $D_{E^{*}}$ on the dual bundle $E^{*}$, etc. In particular we have Bianchi's identity

$$
D_{\text {End } E} \Theta=0 .
$$

If $I$ denotes the identity mapping on $E$, then $c(D)=\operatorname{det}(\kappa \Theta+I)$ is a welldefined differential form whose terms have even degrees, which is called the Chern form of $D$. It is a basic fact that $c(D)$ is a closed form. Moreover its de Rham cohomology class is independent of $D$ and is called the (total) Chern class $c(F)$ of the bundle $F$. 
To prove this, one can consider a smooth one-parameter family $D_{t}$ of connections of $F$ with $D_{0}=D$. If $E^{\prime}$ is the pull-back of $E$ to $X \times[0,1]$, then $D^{\prime}=D_{t}+d_{t}$ is a connection on $E^{\prime}$ and its curvature tensor is

$$
\Theta^{\prime}=\Theta_{t}+d t \wedge \dot{D}_{t}
$$

where $\dot{D}_{t}=d D_{t} / d t$. It is readily checked that it is an element in $\mathscr{E}_{1}(X, \operatorname{End}(F))$. Since $\left(d+d_{t}\right) \operatorname{det}\left(\kappa \Theta^{\prime}+I\right)=0$ we have that

$$
d_{\zeta} \int_{0}^{1} \operatorname{det}\left(\kappa \Theta^{\prime}+I\right)=-\int_{0}^{1} d_{t} \operatorname{det}\left(\Theta^{\prime}+I\right)=c(D)-c\left(D_{1}\right) .
$$

In order to make the computation more explicit we introduce the exterior algebra bundle $\Lambda=\Lambda\left(T^{*}(X) \oplus F \oplus F^{*}\right)$. Any section $\xi \in \mathscr{E}_{k}(X, F)$ corresponds to a section $\tilde{\xi}$ of $\Lambda$; if $\xi=\xi_{1} \otimes e_{1}+\cdots+\xi_{m} \otimes e_{m}$ in a local frame $e_{j}$, then we let $\tilde{\xi}=\xi_{1} \wedge e_{1}+\cdots+\xi_{m} \wedge e_{m}$. In the same way, $a \in \mathscr{E}_{k}(X$, End $E)$ can be identified with

$$
\widetilde{a}=\sum_{j k} a_{j k} \wedge e_{j} \wedge e_{k}^{*},
$$

if $e_{j}^{*}$ is the dual frame, and $a=\sum_{j k} a_{j k} \otimes e_{j} \otimes e_{k}^{*}$ with respect to these frames. A given connection $D=D_{F}$ on $F$ extends in a unique way to a linear mapping $\mathscr{E}(X, \Lambda) \rightarrow \mathscr{E}(X, \Lambda)$ which is a an anti-derivation with respect to the wedge product in $\Lambda$, and such that it acts as the exterior differential $d$ on the $T^{*}(X)$-factor. It is readily seen that

$$
\widetilde{D_{E}} \xi=D \tilde{\xi},
$$

if $\xi$ is a form-valued section of $E$. In the same way we have

Lemma 2.1. If $a \in \mathscr{E}_{k}(X$, End $E)$, then

$$
\widetilde{D_{\text {End }}} a=D \widetilde{a} .
$$

Proof. If $\xi \in \mathscr{E}_{k}(X, E)$ and $\eta \in \mathscr{E}\left(X, E^{*}\right)$, then

$$
D_{\text {End } E}(\xi \otimes \eta)=D_{E} \xi \otimes \eta+(-1)^{k} \xi \otimes D_{E^{*}} \eta,
$$

and thus the snake of $D_{\operatorname{End} E}(\xi \otimes \eta)$ is equal to

$$
\widetilde{D_{E}} \xi \wedge \eta+(-1)^{k+1} \tilde{\xi} \wedge \widetilde{D_{E^{*}} \eta}=D(\tilde{\xi} \wedge \eta)
$$

as claimed. 
Since $D_{\text {End } E} I=0,\left(I=I_{E}\right)$ we have from (2.1) and Lemma 2.1 that

$$
D \widetilde{\Theta}=0 \quad \text { and } \quad D \tilde{I}=0 .
$$

We let $\tilde{I}_{m}=\tilde{I}^{m} / m$ ! and use the same notation for other forms in the sequel. Any form $\omega$ with values in $\Lambda$ can be written $\omega=\omega^{\prime} \wedge \tilde{I}_{m}+\omega^{\prime \prime}$ uniquely, where $\omega^{\prime \prime}$ has lower degree in $e_{j}, e_{k}^{*}$. If we define

$$
\int_{e} \omega=\omega^{\prime}
$$

then this integral is of course linear and moreover

$$
d \int_{e} \omega=\int_{e} D \omega
$$

In fact, since $D \tilde{I}_{m}=0$,

$$
\int_{e} D \omega=\int_{e} d \omega^{\prime} \wedge \tilde{I}_{m}+D \omega^{\prime \prime}=d \omega^{\prime}=d \int_{e} \omega .
$$

Observe that

$$
c(D)=\int_{e}(\kappa \widetilde{\Theta}+\tilde{I})_{m}=\int_{e} e^{\aleph \tilde{\Theta}+\tilde{I}} .
$$

Lemma 2.1 and (2.3) together imply that the Chern form $c(D)$ is closed. Furthermore, following the outline above, we get the formula

$$
d \int_{0}^{1} \int_{e} \aleph \widetilde{\dot{D}} \wedge e^{\aleph \tilde{\Theta}_{t}+\tilde{I}}=c\left(D_{1}\right)-c\left(D_{0}\right),
$$

thus showing that $c\left(D_{0}\right)$ and $c\left(D_{1}\right)$ are cohomologous.

Recall that if the connection $D$ is modified to $D_{1}=D-\gamma$, where $\gamma \in$ $\mathscr{E}_{1}(X$, End $\left.E)\right)$, then $\Theta_{1}=\Theta-D_{\text {End } E} \gamma+\gamma \wedge \gamma$. If we form the explicit homotopy $D_{t}=D-t \gamma$, therefore

$$
\Theta_{t}=\Theta-t D_{\text {End } E} \gamma+t^{2} \gamma \wedge \gamma
$$

and hence, by Lemma 2.1,

$$
\widetilde{\Theta}_{t}=\widetilde{\Theta}-t D \tilde{\gamma}+t^{2} \gamma \widetilde{\wedge} \gamma
$$




\section{Bott-Chern classes}

From now on we assume that $E$ is a holomorphic Hermitian bundle and that $D_{E}$ is the Chern connection and $D_{E}^{\prime}$ is its $(1,0)$-part. Then the induced connection $D_{E^{*}}$ on $E^{*}$ is the Chern connection on $E^{*}$ etc. In particular, our mapping $D$ on $\Lambda$ is of type $(1,0)$, i.e., $D=D^{\prime}+\bar{\partial}$.

Let $E \rightarrow X$ be a Hermitian vector bundle with Chern connection $D_{E}$. The Bott-Chern class $\hat{c}(E)$ is the equivalence class of the Chern form $c\left(D_{E}\right)$ in

$$
\frac{\oplus_{k} \mathscr{E}_{k, k}(X) \cap \operatorname{Ker} d}{\oplus_{k} d d^{c} \mathscr{E}_{k, k}(X)}
$$

LeMma 3.1. Let $D$ be a connection depending smoothly on a real parameter t. Moreover, assume that $L \in \mathscr{E}(X, \operatorname{End}(E))$ depends smoothly on $t$ and that

$$
D_{\text {End } E}^{\prime} L=\dot{D} \text {. }
$$

Also assume that $\Theta_{t}$ has bidegree $(1,1)$ for all $t$. If

$$
v=-\frac{1}{2} \int_{0}^{1} \int_{e} \tilde{L}_{t} \wedge e^{\aleph \tilde{\Theta}_{t}+\tilde{I}} d t
$$

then $-2 \aleph \partial v=b$, where

$$
b=\int_{0}^{1} \int_{e} \aleph \widetilde{\dot{D}}_{t} \wedge e^{\aleph \tilde{\Theta}_{t}+\tilde{I}} d t
$$

This lemma as well as the other material in this section is taken from [8]. However, we use a somewhat different formalism, and for the reader's convenience we supply some simple proofs.

Proof. In view of (2.4) we have that (suppressing the index $t$ )

$$
d \int_{e} \tilde{L} \wedge e^{\aleph \tilde{\Theta}+\tilde{I}}=\int_{e} D \tilde{L} \wedge e^{\aleph \tilde{\Theta}+\tilde{I}}
$$

and by identifying bidegrees we get that

$$
\partial \int_{e} \tilde{L} \wedge e^{\aleph \tilde{\Theta}+\tilde{I}}=\int_{e} D^{\prime} \tilde{L} \wedge e^{\aleph \tilde{\Theta}+\tilde{I}}=\int_{e} \tilde{\dot{D}} \wedge e^{\aleph \tilde{\Theta}+\tilde{I}} .
$$

Since $d b=c\left(D_{1}\right)-c\left(D_{0}\right)$, cf., (2.6), we thus have

$$
-d d^{c} v=c\left(D_{1}\right)-c\left(D_{0}\right) .
$$


By deforming the metric one can use this lemma to show that $\hat{c}(E)$ is independent of the Hermitian structure on $E$, see [8]. However we are interested in a somewhat different situation. Assume that we have the short exact sequence of Hermitian vector bundles

$$
0 \longrightarrow S \stackrel{j}{\longrightarrow} E \stackrel{g}{\longrightarrow} Q \longrightarrow 0,
$$

where $Q$ and $S$ are equipped with the metrics induced by the Hermitian metric of $E$. Then

$$
j^{*} \oplus g: E \rightarrow S \oplus Q
$$

is a smooth vector bundle isomorphism. If $D_{S}$ and $D_{Q}$ are the Chern connections on $S$ and $Q$ respectively, then

$$
D_{E} \sim\left(\begin{array}{cc}
D_{S} & -\beta^{*} \\
\beta & D_{Q}
\end{array}\right)
$$

with respect to the isomorphism (3.4), where $\beta \in \mathscr{E}_{1,0}(X, \operatorname{Hom}(S, Q))$ is the second fundamental form, see [10]. We shall now modify the connection $D=$ $D_{E}$ to $D_{b}=D-\gamma_{b}$, where $\gamma_{b}=D_{\text {End } E}^{\prime} j j^{*}$. It turns out that $\gamma=g^{*} \circ \beta \circ j^{*}$, thus $\gamma \wedge \gamma=0$, and that $D_{\text {End } E} \gamma=\bar{\partial} \gamma$. Moreover, it follows that

$$
D_{b} \sim\left(\begin{array}{cc}
D_{S} & * \\
0 & D_{Q}
\end{array}\right)
$$

and hence

$$
\Theta_{b} \sim\left(\begin{array}{cc}
\Theta_{S} & * \\
0 & \Theta_{Q}
\end{array}\right)
$$

so that $c\left(D_{b}\right)=c\left(D_{S}\right) c\left(D_{Q}\right)$. If $D_{t}=D-t \gamma_{b}$ we have $\Theta_{t}=\Theta-t \bar{\partial} \gamma_{b}$; thus it has bidegree $(1,1)$. If we let

$$
b=\int_{0}^{1} \int_{e} \kappa \tilde{\gamma}_{b} \wedge e^{\tilde{I}+\kappa \tilde{\Theta}-t \aleph \bar{\partial} \tilde{\gamma}_{b}}=\sum_{\ell \geq 0} \int_{e} \aleph \tilde{\gamma}_{b} \wedge e^{\tilde{I}+\kappa \tilde{\Theta}} \wedge \frac{1}{(\ell+1) !}\left(-\aleph \bar{\partial} \tilde{\gamma}_{b}\right)^{\ell}
$$

it follows from (2.6) that $d b=c\left(D_{E}\right)-c\left(D_{S}\right) c\left(D_{Q}\right)$. Moreover, if $L=$ $j j^{*} /(1-t)$, then (3.1) holds. In fact, $\dot{D}=-\gamma_{b}$, and $\left[j j^{*}, g^{*} \circ \beta \circ j^{*}\right]=$ $g^{*} \circ \beta \circ j^{*}$, so that

$$
D_{\text {End } E, t}^{\prime} L=D_{\text {End } E}^{\prime} L-t\left[\gamma_{b}, L\right]=\frac{1}{1-t} \gamma_{b}-\frac{t}{1-t} \gamma_{b}=\gamma_{b} .
$$


Proposition 3.2. If

$$
v=\sum_{\ell=1}^{m-1} \frac{(-1)^{\ell}}{2 \ell} \int_{e} \widetilde{j j^{*}} \wedge\left(\tilde{I}+\kappa \tilde{\Theta}-\kappa \bar{\partial} \tilde{\gamma}_{b}\right)_{m-\ell-1} \wedge\left(-\aleph \bar{\partial} \tilde{\gamma}_{b}\right)_{\ell},
$$

then $-2 \aleph \partial v=b$.

Proof. Observe that

$$
\partial \int_{0}^{1-\epsilon} \int_{e} \frac{\widetilde{j j^{*}}}{1-t} \wedge e^{\tilde{I}+\aleph \Theta_{1}} d t=\int_{0}^{1-\epsilon} \int_{e} \frac{D_{1} \widetilde{j j^{*}}}{1-t} \wedge e^{\tilde{I}+\kappa \Theta_{1}} d t=0,
$$

since $D_{1} \widetilde{j j^{*}}=D_{\text {End } E, 1} \widetilde{j} j^{*}=0$ in view of Lemma 2.1 and (3.8). Therefore,

$\aleph \partial \int_{0}^{1-\epsilon} \int_{e} \tilde{j j^{*}} \wedge \frac{e^{\tilde{I}+\aleph \tilde{\Theta}-t \aleph \bar{\partial} \tilde{\gamma}_{b}}-e^{\tilde{I}+\aleph \tilde{\Theta}-\aleph \bar{\partial} \tilde{\gamma}_{b}}}{1-t} d t=\int_{0}^{1-\epsilon} \int_{e} \aleph \tilde{\gamma}_{b} \wedge e^{\tilde{I}+\aleph \tilde{\Theta}-t \aleph \bar{\partial} \tilde{\gamma}_{b}}$.

The proposition now follows by letting $\epsilon \rightarrow 0$ and computing the $t$-integral on the left hand side.

Altogether we therefore have that $-d d^{c} v=c\left(D_{E}\right)-c\left(D_{S}\right) c\left(D_{Q}\right)$ and thus $\hat{c}(E)=\hat{c}(S) \hat{c}(Q)$.

\section{Proof of the main formula}

Let $f$ be a nontrivial holomorphic section of $E, Z=\{f=0\}$, and let $S$ be the trivial subbundle of $E$ over $X \backslash Z$, generated by the $f$. We then have the short exact sequence (3.3) over $X \backslash Z$, where $g: E \rightarrow Q=E / Q$ is the natural projection. Let $\sigma$ be the section of the dual bundle $E^{*}$ with minimal norm such $\sigma \cdot f=1$. Then clearly

$$
\widetilde{j j^{*}}=f \wedge \sigma
$$

Observe that the natural conjugate-linear isometry $E \simeq E^{*}, \eta \mapsto \eta^{*}$, defined by

$$
\eta^{*} \cdot \xi=\langle\xi, \eta\rangle, \quad \xi \in \mathscr{E}(X, E),
$$

extends to an isometry on the space of form-valued sections.

Lemma 4.1. If $\phi=-\partial \log |f|^{2}$, then $D^{\prime} \sigma=\phi \wedge \sigma$.

Proof. Observe that $\sigma=f^{*} /|f|^{2}$. Since $D=D_{E}$ is the Chern connection, $D^{\prime} f^{*}=(\bar{\partial} f)^{*}=0$, so we have

$$
D^{\prime} \sigma=D^{\prime}\left(f^{*} /|f|^{2}\right)=\partial \frac{1}{|f|^{2}} \wedge f^{*}=-\partial \log |f|^{2} \wedge \sigma .
$$


Following Section 3 we let $\gamma_{b}=D_{\text {End } E}^{\prime}\left(j j^{*}\right)$. By Lemma 4.1 and (4.1) we then have

$$
\tilde{\gamma}_{b}=(D f-f \wedge \phi) \wedge \sigma
$$

and

$$
\bar{\partial} \tilde{\gamma}_{b}=(D f-f \wedge \phi) \wedge \bar{\partial} \sigma+(\Theta f+f \wedge \bar{\partial} \phi) \wedge \sigma .
$$

The following formula is the key point in the analysis of the singularities of $c\left(D_{Q}\right)$.

Proposition 4.2. In $X \backslash Z$ we have the explicit formula

$$
c\left(D_{Q}\right)=\int_{e} f \wedge \sigma \wedge e^{\tilde{I}+\kappa \tilde{\Theta}-\aleph D f \wedge \bar{\partial} \sigma} .
$$

Proof. Since $\Theta_{b}=\Theta-\bar{\partial} \gamma_{b}$ we have by (4.3) that

$$
\widetilde{\Theta}_{b}=\widetilde{\Theta}-((D f-f \wedge \phi) \wedge \bar{\partial} \sigma+(\Theta f+f \wedge \bar{\partial} \phi) \wedge \sigma) .
$$

For any section $A$ of $\operatorname{End}(E)$,

$$
\int_{e} f \wedge \sigma \wedge \tilde{A}_{m-1}=\int_{e} f \wedge \sigma \wedge e^{\tilde{A}}
$$

is the determinant of the restriction of $A$ to $Q$, that is, the determinant of $g \mathrm{Ag}^{*}$. In view of (3.6) therefore the expression on the right hand side of (4.4) is equal to $\operatorname{det}\left(I_{Q}+\kappa \Theta_{Q}\right)=c\left(D_{Q}\right)$.

Now, let $v$ and $b$ be the forms in $X \backslash Z$ defined by (3.7) and (3.9).

Proposition 4.3. (i) The forms $v, b, c\left(D_{Q}\right)$, and $c\left(D_{S}\right) \wedge c\left(D_{Q}\right)$ are locally integrable in $X$.

(ii) If the natural extensions are denoted by capitals, then

$$
-2 \aleph \partial V=B,
$$

and

$$
-d d^{c} V=c\left(D_{E}\right)-C\left(D_{S}\right) C\left(D_{Q}\right) .
$$

Proof. This is clearly a local question at $Z$. Locally we can write $f=$ $f_{1} e_{j}+\cdots+f_{m} e_{m}$, where $e_{j}$ is a local holomorphic frame for $E$. In a small neighborhood $U$ of a given point in $X$, Hironaka's theorem provides an $n$ dimensional complex manifold $\widetilde{U}$ and a proper mapping $\Pi: \widetilde{U} \rightarrow U$ which is 
a biholomorphism outside $\Pi^{-1}\left(\left\{f_{1} \cdots f_{v}=0\right\}\right)$, and such that locally on $\widetilde{U}$ there are holomorphic coordinates $\tau$ such that $\Pi^{*} f_{j}=u^{j} \tau_{1}^{\alpha-1} \cdots \tau_{n}^{\alpha_{n}}$, where $u_{j}$ nonvanishing; i.e., roughly speaking $\Pi^{*} f_{j}$ are monomials. By a resolution over a suitable toric manifold, following [3] and [18], we may assume in the same way that one of the functions so obtained divides the other ones. For simplicity we will make a slight abuse of notation and suppress all occurring $\Pi^{*}$ and thus denote these functions by $f_{j}$ as well. We may therefore assume that $f=f_{0} f^{\prime}$ where $f_{0}$ is a holomorphic function and $f^{\prime}$ is a non-vanishing section. Since $\sigma=f^{*} /|f|^{2}$, it follows that $\sigma=\sigma^{\prime} / f_{0}$ where $\sigma^{\prime}$ is smooth, and hence

$$
\widetilde{j j^{*}}=f \wedge \sigma=f^{\prime} \wedge \sigma^{\prime}
$$

is smooth in this resolution. Moreover, $D f \wedge \bar{\partial} \sigma=D f^{\prime} \wedge \bar{\partial} \sigma^{\prime}+\cdots$, where $\cdots$ denote terms that contain some factor $f^{\prime}$ or $\sigma^{\prime}$. In view of Proposition 4.2 it follows that (the pullback of) $c\left(D_{Q}\right)$ is smooth, and therefore locally integrable. Since the push-forward of a locally integrable form is locally integrable we can conclude that $c\left(D_{Q}\right)$ is locally integrable.

It follows that also $\tilde{\gamma}_{b}=D^{\prime}(f \wedge \sigma)$ and $\bar{\partial} \tilde{\gamma}_{b}$ are smooth. Since (4.6) and (4.7) hold in $X \backslash Z$ and $c\left(D_{E}\right)$ is smooth, it follows that all the forms are smooth in the resolution. We can conclude that all the forms are locally integrable in $X$ and that (4.6) and (4.7) hold.

The presence of the factor $\widetilde{j j^{*}}=f \wedge \sigma$ implies that, cf., (3.9),

$$
v=\sum_{\ell=1}^{m-1} \frac{(-1)^{\ell}}{2 \ell} \int_{e} f \wedge \sigma \wedge(\tilde{I}+\kappa \tilde{\Theta}-\aleph D f \wedge \bar{\partial} \sigma)_{m-1-\ell} \wedge(-\aleph D f \wedge \bar{\partial} \sigma)_{\ell} .
$$

Definition 4.4. We define the current $W$ as

$$
\begin{gathered}
W=\log (1 /|f|) c\left(D_{Q}\right)-V \\
=\log (1 /|f|) \int_{e} f \wedge \sigma \wedge(\aleph \tilde{\Theta}+\tilde{I}-\aleph D f \wedge \bar{\partial} \sigma)_{m-1} \\
-\sum_{\ell=1}^{m-1} \frac{(-1)^{\ell}}{2 \ell} \int_{e} f \wedge \sigma \wedge(\tilde{I}+\kappa \tilde{\Theta}-\aleph D f \wedge \bar{\partial} \sigma)_{m-1-\ell} \wedge(-\aleph D f \wedge \bar{\partial} \sigma)_{\ell} .
\end{gathered}
$$

In particular, if $E$ is a line bundle, i.e., $m=1$, then $V=0$, and since $\sigma \cdot f=1$ we have that $W=\log (1 /|f|)$. It is now a simple matter to conclude the proof of Theorem 1.1.

Proof of Theorem 1.1. Consider a resolution of singularities in which $f=f_{0} f^{\prime}$ with $f^{\prime}$ non-vanishing, as in the proof of Proposition 4.3. Then we 
know that $c\left(D_{Q}\right)$ is smooth, and therefore $\log |f| c\left(D_{Q}\right)$ is locally integrable there. Moreover, since $\log |f|=\log \left|f_{0}\right|+\log \left|f^{\prime}\right|$ we have that

$$
\begin{aligned}
& \lambda|f|^{2 \lambda} \frac{\aleph \partial|f|^{2} \wedge \bar{\partial}|f|^{2}}{|f|^{4}} \wedge c\left(D_{Q}\right) \\
& \quad=\lambda\left|f_{0}\right|^{2 \lambda}\left|f^{\prime}\right|^{2 \lambda} \aleph\left(\frac{d f_{0}}{f_{0}}+\frac{\partial\left|f^{\prime}\right|^{2}}{\left|f^{\prime}\right|^{2}}\right) \wedge\left(\frac{d \bar{f}_{0}}{\bar{f}_{0}}+\frac{\bar{\partial}\left|f^{\prime}\right|^{2}}{\left|f^{\prime}\right|^{2}}\right) \wedge c\left(D_{Q}\right) .
\end{aligned}
$$

This form is locally integrable for $\lambda>0$ and tends to

$$
\left[f_{0}=0\right] \wedge c\left(D_{Q}\right)=d d^{c}\left(\log |f| c\left(D_{Q}\right)\right) \mathbf{1}_{\left\{f_{0}=0\right\}}
$$

when $\lambda \rightarrow 0$, where $\left[f_{0}=0\right]$ is the current of integration over the divisior defined by $f_{0}$. Thus $M$ is a closed current of bidegree $(*, *)$ and order zero in $X$ with support on $Z$. Thus, see, e.g., [10], $M_{k}=0$ for $k<p=\operatorname{codim} Z$ and $M_{p}=\sum_{j} \alpha_{j} Z_{j}^{p}$ for some numbers $\alpha_{j}$. To see that $\alpha_{j}$ is precisely the multiplicity of $f$ on $Z_{j}^{p}$ we can locally deform the Hermitian metric to a trivial metric. Then $\Theta=0$ and a straight-forward computation, see [2], reveals that $c_{p-1}\left(D_{Q}\right)=\left(d d^{c} \log |f|\right)^{p-1}$. Therefore, $M=d d^{c}\left(\log |f|\left(d d^{c} \log |f|\right)^{p-1}\right)$ which is equal to the multiplicity times $\left[Z_{j}^{p}\right]$ according to King's formula, see [11] and [10]. Thus part (i) of the theorem is proved. Since $c\left(D_{S}\right)-1=$ $c_{1}\left(D_{S}\right)=d d^{c} \log (1 /|f|)$ we have

$d d^{c}\left(\log (1 /|f|) c\left(D_{Q}\right)\right)=C\left(D_{S}\right) \wedge C\left(D_{Q}\right)-C\left(D_{Q}\right)-d d^{c}\left(\log |f| c\left(D_{Q}\right)\right) \mathbf{1}_{Z}$.

Now part (ii) follows from Proposition 4.3, cf, (4.9).

\section{A direct approach to (1.6)}

We use the same notation as in the previous section. In [6], Berndtsson introduced the deformation $D_{a}=D-\gamma_{a}$ of $D$ on $E$, where

$$
\tilde{\gamma}_{a}=D f \wedge \sigma,
$$

in order to construct Koppelman formulas for $\bar{\partial}$ on manifolds. He proved formula (5.7) below for $k=m$ (i.e., $\bar{\partial} a_{m}=d a_{m}=c_{m}(E)$ ). For the general case first we must understand the geometric meaning of $D_{a}$. Since $D_{a} f=0$, we have that $D_{a} \xi$ is in $S$ if $\xi$ is a section of $S$. Moreover, if $\xi$ is a section of $S^{\perp}$, then $D_{a} \xi=D_{E} \xi$. Now

$$
g \xi \mapsto g\left(D_{a} \xi\right)
$$


is a well-defined connection on $Q$, and we claim that it is actually the Chern connection $D_{Q}$. In fact, if $\eta=g \xi$, then

$$
D_{Q} \eta=g\left(D_{E}\left(g^{*} \eta\right)\right)=g\left(D_{a}\left(g^{*} \eta\right)\right)=g\left(D_{a} \xi\right) .
$$

It follows that $\Theta_{Q} \eta=g\left(\Theta_{a} \xi\right)$, and since $\Theta_{a} \xi=0$ if $\xi$ takes values in $S$, we have that

$$
\kappa \Theta_{a} \sim\left(\begin{array}{cc}
0 & * \\
0 & \kappa \Theta_{Q}
\end{array}\right)
$$

with respect to the smooth isomorphism (3.4). Therefore,

$$
\aleph \Theta_{a}+I_{E} \sim\left(\begin{array}{cc}
I_{S} & * \\
0 & I_{Q}+\kappa \Theta_{Q},
\end{array}\right),
$$

and taking the determinant, we find that

$$
c\left(D_{Q}\right)=c\left(D_{a}\right) .
$$

Proposition 5.1. If $\gamma_{a}$ is defined by (5.1), then

(5.5) $-t D \tilde{\gamma}_{a}+t^{2} \gamma_{a} \widetilde{\wedge} \gamma_{a}=-t(D f \wedge \bar{\partial} \sigma+\Theta f \wedge \sigma)+\left(t-t^{2}\right) D f \wedge \phi \wedge \sigma$.

Proof. A simple computation yields

$$
D \tilde{\gamma}_{a}=\Theta f \wedge \sigma+D f \wedge \bar{\partial} \sigma+D f \wedge \phi \wedge \sigma
$$

and

$$
\gamma_{a} \widetilde{\wedge} \gamma_{a}=D f \wedge \sigma \cdot D f \wedge \sigma,
$$

where the dot means the natural contraction of $E$ and $E^{*}$ so that $\xi \cdot(\alpha \wedge \eta)=$ $\alpha(\xi \cdot \eta)$ if $\xi$ and $\eta$ are sections of $E$ and $E^{*}$, respectively, and $\alpha$ is a form. Since $\sigma \cdot D f=-D^{\prime} \sigma \cdot f=\phi$ we get the desired formula.

Proposition 5.2. If

$$
a=\int_{e} \aleph D f \wedge \sigma \wedge e^{\tilde{I}+\aleph \tilde{\Theta}} \wedge \sum_{\ell=0}^{\infty} \frac{(-\aleph D f \wedge \bar{\partial} \sigma)^{\ell}}{(\ell+1) !}
$$

then

$$
\bar{\partial} a=d a=c\left(D_{E}\right)-c\left(D_{Q}\right)
$$

in $X \backslash Z$. 
Proof. We choose the homotopy $D_{t}=D-t \gamma_{a}$ between $D=D_{0}$ and $D_{1}=D_{a}$.

In view of (2.6), (2.1), and Proposition 5.1 we have that

$$
a=\int_{e} \int_{0}^{1} \aleph D f \wedge \sigma \wedge e^{\tilde{I}+\aleph \tilde{\Theta}-t \aleph(\Theta f \wedge \sigma+D f \wedge \bar{\partial} \sigma)-\left(t-t^{2}\right) D f \wedge \phi \wedge \sigma} d t
$$

satisfies the second equality in (5.7) in $X \backslash Z$. Noticing that $\sigma \wedge \sigma=0$, a computation of the $t$-integral yields (5.6). Since $a$ has bidegree $(*, *-1)$ and $d a$ has bidegree $(*, *)$ it follows that $\bar{\partial} a=d a$.

The forms $a$ and $b$ are related in the following way.

Proposition 5.3. In $X \backslash Z$ we have that

$$
b=a+\kappa \partial \log |f|^{2} \wedge c\left(D_{Q}\right)
$$

Proof. Starting with (3.7) we have

$$
\begin{aligned}
b & =\int_{e} \aleph(D f-f \wedge \phi) \wedge \sigma \wedge e^{\tilde{I}+\aleph \tilde{\Theta}} \wedge \sum_{\ell=0}^{\infty} \frac{(-\aleph D f+\aleph f \wedge \phi)^{\ell}}{(1+\ell) !} \wedge(\bar{\partial} \sigma)^{\ell} \\
& =-\int_{\ell} e^{\tilde{I}+\kappa \tilde{\Theta}} \wedge \sum_{\ell=0}^{\infty} \frac{(-\aleph D f+\aleph f \wedge \phi)^{\ell+1}}{(\ell+1) !} \wedge \sigma \wedge(\bar{\partial} \sigma)^{\ell} \\
& =-\int_{\ell} e^{\tilde{I}+\kappa \tilde{\Theta}-\aleph D f+\aleph f \wedge \phi} \wedge \sum_{\ell=0}^{\infty} \sigma \wedge(\bar{\partial} \sigma)^{\ell} \\
& =-\int_{\ell} e^{\tilde{I}+\kappa \tilde{\Theta}-\aleph D f} \wedge(1+\aleph f \wedge \phi) \wedge \sum_{\ell} \sigma \wedge(\bar{\partial} \sigma)^{\ell} .
\end{aligned}
$$

In view of (6.3) and (6.6), recalling that $\phi=-\partial \log |f|^{2}$, we now get (5.8).

By a resolution of singularities as in the proof of Proposition 4.3 above one can see that $a$ is locally integrable. Let $A$ denote its natural extension. By such a resolution one can also verify that the formal computation (using Proposition 5.3) $-2 \aleph \partial\left(\log (1 /|f|) c\left(D_{Q}\right)-V\right)=B-\aleph \partial \log |f|^{2} \wedge C\left(D_{Q}\right)=A$ is ligitimate, and thus we have

$$
A=-2 \ltimes \partial W .
$$

As a consequence we get that $\bar{\partial} A=d A=c\left(D_{E}\right)-c\left(D_{Q}\right)-M$. 


\section{Factorization of currents}

Since $a$ and $c\left(D_{Q}\right)$ are locally integrable, $|f|^{2 \lambda} a$ and $|f|^{2 \lambda} c\left(D_{Q}\right)$ are welldefined currents for $\operatorname{Re} \lambda>-\epsilon$ and we have

$$
A=\left.|f|^{2 \lambda} a\right|_{\lambda=0} \quad \text { and } \quad C\left(D_{Q}\right)=\left.|f|^{2 \lambda} c\left(D_{Q}\right)\right|_{\lambda=0} .
$$

It also follows that

$$
M=-\left.d|f|^{2 \lambda} \wedge a\right|_{\lambda=0}=-\left.\bar{\partial}|f|^{2 \lambda} \wedge a\right|_{\lambda=0} .
$$

Now consider the expression (5.6) for $a$. Since each term in $\exp (\tilde{I}+\kappa \tilde{\Theta})$ has the same degree in $e_{j}$ and $e_{k}^{*}$ it must be multiplied by terms with the same property in order to get a product with full degree. Therefore we can rewrite $a$ as

$$
a=-\int_{e} e^{\tilde{I}+\aleph \tilde{\Theta}-\aleph D f} \wedge \sum_{0}^{\infty} \sigma \wedge(\bar{\partial} \sigma)^{\ell} .
$$

In [1] we introduced the currents

$$
U=\left.|f|^{2 \lambda} \frac{\sigma}{1-\bar{\partial} \sigma}\right|_{\lambda=0}=\left.|f|^{2 \lambda} \wedge \sigma \wedge \sum_{\ell}(\bar{\partial} \sigma)^{\ell-1}\right|_{\lambda=0}
$$

and

$$
R=\left.\bar{\partial}|f|^{2 \lambda} \wedge \frac{\sigma}{1-\bar{\partial} \sigma}\right|_{\lambda=0}=\left.\bar{\partial}|f|^{2 \lambda} \wedge \sigma \wedge \sum_{\ell}(\bar{\partial} \sigma)^{\ell-1}\right|_{\lambda=0} .
$$

It is part of the statement that the right hand sides are current valued holomorphic functions for $\lambda>-\epsilon$, evaluated at $\lambda=0$. In general $U$ and $R$ are not locally integrable. The current $R$ is supported on $Z$,

$$
R=R_{p}+\cdots+R_{\min (m, n)},
$$

where $R_{k}$ is the component of bidegree $(0, k)$ taking values in $\Lambda^{k} E^{*}$, and $\left(\delta_{f}-\bar{\partial}\right) U=1-R$. In view of (6.3), (6.1), and (6.2) we have the factorization formulas

$$
\begin{aligned}
M & =\int_{e} e^{\aleph \widetilde{\Theta}+\tilde{I}-\aleph D f} \wedge R, \\
A & =-\int_{e} e^{\aleph \widetilde{\Theta}+\tilde{I}-\aleph D f} \wedge U,
\end{aligned}
$$


and moreover, cf. (4.4),

$$
C\left(D_{Q}\right)=\int_{e} f \wedge \sigma \wedge e^{\aleph \tilde{\Theta}+\tilde{I}-\aleph D f \wedge \bar{\partial} \sigma}=\int_{e} e^{\aleph \tilde{\Theta}+\tilde{I}-\aleph D f} \wedge f \wedge U .
$$

\section{Positivity}

Let $E \rightarrow X$ be a Hermitian holomorphic bundle as before and let $e_{j}$ be an orthonormal local frame. A section

$$
A=i \sum_{j k} A_{j k} \otimes e_{j} \otimes e_{k}^{*}
$$

of $T_{1,1}^{*}(X) \otimes \operatorname{End}(E)$ is Hermitian if $A_{j k}=-\overline{A_{k j}}$. It then induces a Hermitian form $a$ on $T^{1,0}(X) \otimes E^{*}$ by

$$
a\left(\xi \otimes e_{j}^{*}, \eta \otimes e_{k}^{*}\right)=A_{j k}(\xi, \bar{\eta}),
$$

if $\xi, \eta$ are $(1,0)$-vectors. We say that $A$ is (Bott-Chern) positive, $A \geq_{B} 0$, if the form $a$ is positively semi-definite. In the same way any Hermitian $A$ induces a Hermitian form $a^{\prime}$ on $T^{1,0}(X) \otimes E$ and it is called Nakano positive, $A \geq_{N} 0$, if $a^{\prime}$ is positively semi-definite.

Notice that $\aleph \Theta$ is Hermitian; it is said to be Nakano positive if $\kappa \Theta \geq_{N} 0$. Analogously we say that $E$ is positive, $E \geq_{B} 0$, if $\aleph \Theta \geq_{B} 0$. Neither of these positivity concepts implies the other one unless $m=1$.

Since $\Theta_{j k}\left(E^{*}\right)=-\Theta_{j k}(E)$ it follows that $E$ is positive in our sense if and only if $E^{*}$ is Nakano negative. The next proposition explains the interest of Bott-Chern positivity in this context.

Proposition 7.1. Let

$$
0 \rightarrow S \rightarrow E \rightarrow Q \rightarrow 0
$$

be a short exact sequence of Hermitian holomorphic vector bundles. Then $E \geq_{B} 0$ implies that $Q \geq_{B} 0$.

Proof. It is well-known, see for instance [10], that $E \leq_{N} 0$ implies that $S \leq_{N} 0$. From the sequence (7.1) above we get the exact sequence $0 \rightarrow Q^{*} \rightarrow$ $E^{*} \rightarrow S^{*} \rightarrow 0$. Since $E^{*} \leq_{N} 0$ implies $Q^{*} \leq_{N} 0$, it follows that $E \geq_{B} 0$ implies $Q \geq_{B} 0$.

The next simple lemma reveals that our definition of Bott-Chern positivity coincides with the one used in [8]. 
Lemma 7.2. $A \geq_{B} 0$ if and only if there are sections $f_{\ell}$ of $T_{1,0}^{*}(X) \otimes E$ such that

$$
A=i \sum_{\ell} f_{\ell} \otimes f_{\ell}^{*}
$$

Observe that if $f_{\ell}=\sum f_{j}^{\ell} \otimes e_{j}$, then $f_{\ell}^{*}=\sum \bar{f}_{j}^{\ell} \otimes e_{j}^{*}$ since $e_{j}$ is ortonormal.

Proof. If (7.2) holds, then

$$
a(\xi, \xi)=\sum_{\ell} f_{\ell}(\xi) f_{\ell}^{*}\left(\xi^{*}\right)=\sum\left|f_{\ell}(\xi)\right|^{2} \geq 0
$$

for all $\xi$ in $T^{1,0} \otimes E^{*}$. Conversely, if $a$ is positive, it is diagonalizable, and so there is a basis $f_{\ell}$ for $T_{1,0}^{*} \otimes E$ such that (7.2) holds.

If we identify $f_{\ell}$ with $\sum f_{j}^{\ell} \wedge e_{j}$ as before, then (7.2) means that

$$
\tilde{A}=-i \sum_{\ell} f_{\ell} \wedge f_{\ell}^{*}
$$

If $B=\sum B_{j k} e_{j} \otimes e_{j}^{*}$ is a scalar-valued section of End $E$, then it is Hermitian if and only if $B_{j k}=\bar{B}_{k j}$ and it is positively semi-definite if and only if

$$
B=\sum_{\ell} g_{\ell} \otimes g_{\ell}^{*}
$$

for some sections $g_{\ell}$ of $E$; or equivalently,

$$
\tilde{B}=\sum_{\ell} g_{\ell} \wedge g_{\ell}^{*}
$$

Proposition 7.3. Assume that $A_{j}$ are $(1,1)$-form-valued Hermitian sections of $E$ and $B_{k}$ scalarvalued sections, such that $A_{j} \geq_{B} 0$ and $B_{k} \geq 0$. Then

$$
\int_{e} \tilde{A}_{1} \wedge \ldots \wedge \tilde{A}_{r} \wedge \tilde{B}_{r+1} \wedge \ldots \wedge \tilde{B}_{m}
$$

is a positive $(r, r)$-form. 
Proof. In view of (7.3) and (7.4), we see that (7.5) is a sum of terms like

$$
\begin{gathered}
\int_{e}(-i)^{r} f_{1} \wedge f_{1}^{*} \wedge \ldots \wedge f_{r} \wedge f_{r}^{*} \wedge g_{r+1} \wedge g_{r+1}^{*} \wedge \ldots \wedge g_{m} \wedge g_{m}^{*} \\
=(-i)^{r} c_{m-r} \int_{e} f_{1} \wedge \ldots f_{r} \wedge \ldots g_{m} \wedge f_{1}^{*} \wedge \ldots \wedge f_{r}^{*} \wedge \ldots g_{m}^{*} \\
=(-i)^{r} c_{m-r} \int_{e} \omega \wedge e_{1} \wedge \ldots \wedge e_{m} \wedge \bar{\omega} \wedge e_{1}^{*} \wedge \ldots \wedge e_{m}^{*}
\end{gathered}
$$

where $\omega$ is an $(r, 0)$-form and $c_{p}=(-1)^{p(p-1) / 2}=i^{p(p-1)}$. By further simple computations,

$$
\begin{aligned}
(-i)^{r} c_{m-r}(-1)^{m r} \int_{e} \omega \wedge \bar{\omega} \wedge e_{1} \wedge & \ldots \wedge e_{m} \wedge e_{1}^{*} \wedge \ldots \wedge e_{m}^{*} \\
& =(-i)^{r} c_{m-r}(-1)^{m r} c_{m} \omega \wedge \bar{\omega}=i^{r^{2}} \omega \wedge \bar{\omega},
\end{aligned}
$$

the proposition follows, since the last form is positive.

Proposition 7.4. If $E \geq_{B} 0$ (or $E \geq_{N} 0$ ), then the Chern forms $c_{k}\left(D_{E}\right)$ are positive for all $k$.

Proof. Since $\alpha \Theta \geq_{B} 0$ by assumption, and clearly $I \geq 0$, it follows from Proposition 7.3 that

$$
c_{k}\left(D_{E}\right)=\int_{e}(\aleph \widetilde{\Theta})_{k} \wedge \tilde{I}_{m-k}
$$

is positive.

Proof of Theorem 1.2. We have just seen that $c\left(D_{E}\right) \geq 0$. From (1.3) it follows that the current $M_{k}$ is positive if $c_{k-1}\left(D_{Q}\right)$ is positive. From (4.4) we have that

$$
\begin{aligned}
c_{k-1}\left(D_{Q}\right) & =\int_{e} f \wedge \sigma \wedge(\aleph \widetilde{\Theta}-\aleph D f \wedge \bar{\partial} \sigma)_{k-1} \wedge \tilde{I}_{m-k} \\
& =\sum_{j=1}^{k-1} \int_{e} f \wedge \sigma \wedge(\kappa \widetilde{\Theta})_{k-1-j} \wedge(-\aleph D f \wedge \bar{\partial} \sigma)_{j} \wedge \tilde{I}_{m-k}
\end{aligned}
$$

If $s=f^{*}$ as before, then $\sigma=s /|f|^{2}$, and therefore we have

$$
c_{k-1}\left(D_{Q}\right)=\sum_{j=1}^{k-1} \int_{e} \frac{f \wedge s}{|f|^{2}} \wedge\left(\frac{-\aleph D f \wedge \bar{\partial} s}{|f|^{2}}\right)_{j} \wedge(\aleph \widetilde{\Theta})_{k-1-j} \wedge \tilde{I}_{m-k}
$$

Since $\bar{\partial} s=(D f)^{*}$ it now follows immediately from Proposition 7.3 that $c_{k}\left(D_{Q}\right)$ is positive if $\kappa \Theta \geq_{B} 0$. 
It remains to see that one can choose $W$ so that it is positive where $|f|<1$. Notice that if some of the $A_{j}$ in (7.5) are replaced by $A_{j}^{\prime} \geq_{B} A_{j}$, then the resulting form will be larger; this follows immediately from the proof. Now, $\log (1 /|f|) c\left(D_{Q}\right)$ is positive when $|f|<1$. From (4.8) we have that $v_{k}=\sum_{\ell=1}^{k} \frac{(-1)^{\ell}}{2 \ell} \int_{e} f \wedge \sigma \wedge(\aleph \widetilde{\Theta}-\aleph D f \wedge \bar{\partial} \sigma)_{k-\ell} \wedge(-\aleph D f \wedge \bar{\partial} \sigma)_{\ell} \wedge \tilde{I}_{m-k-1}$.

Since this is an alternating sum of positive terms it has no sign. If we replace each factor $-\aleph D f \wedge \bar{\partial} \sigma$ by $\aleph \widetilde{\Theta}-\aleph D f \wedge \bar{\partial} \sigma$, then we get a larger form which in addition is closed, since it is just a certain constant times $c_{k}\left(D_{Q}\right)$, cf., (7.6). Therefore, for a suitable constant $v_{k}-v_{k}^{\prime}=-v_{k}+v_{k} c_{k}\left(D_{Q}\right)$ is a positive form and $d v_{k}^{\prime}=d v_{k}$. Thus the current

$$
W_{k}^{\prime}=-V_{k}+v_{k} C_{k}\left(D_{Q}\right)+\log (1 /|f|) C_{k}\left(D_{Q}\right)
$$

will have the stated property.

The modification of $v$ in last part of the proof is precisely as in [8] but with our notation, and for an arbitrary $k$ rather than just $k=m-1$. It is not necessary to consider each $v_{k}$ separately. By the same argument one can see directly that $-v^{\prime}=-v+v c\left(D_{Q}\right)$ is positive if $v$ is appropriately chosen, and $d v^{\prime}=d v$.

One can prove that if we multiply (7.7) with $\lambda \partial|f|^{2} \wedge \bar{\partial}|f|^{2} /|f|^{2}$ and let $\lambda \rightarrow 0^{+}$, then all terms with $j<p-1$ will disappear; see for instance the proof of Theorem 1.1 in [1]. We thus have

Proposition 7.5. If $p=\operatorname{codim}\{f=0\}$, then

$$
\begin{aligned}
M_{k}=\lim _{\lambda \rightarrow 0^{+}} \lambda|f|^{2 \lambda} & \stackrel{\partial|f|^{2} \wedge \bar{\partial}|f|^{2}}{|f|^{2}} \\
& \wedge \sum_{j=p-1}^{k-1} \int_{e} \frac{f \wedge s}{|f|^{2}} \wedge\left(\frac{-\aleph D f \wedge \bar{\partial} s}{|f|^{2}}\right)_{j} \wedge(\aleph \widetilde{\Theta})_{k-1-j} \wedge \tilde{I}_{m-k} .
\end{aligned}
$$

From this formula it is apparent that $M_{k}$ vanishes if $k<p$, and that $M_{p}$ is positive, regardless of $\aleph \Theta$. One can also derive this formula from (6.4).

Remark 2. When $k>p, M_{k}$ depends on the metric, but there is still a certain uniqueness: Let $Z^{k}$ be the union of the irreducible components $Z_{j}^{k}$ of $Z$ of codimension $k$. One can verify, see [2], that the restriction of $M_{k}$ to $Z^{k}$ is a sum

$$
\sum_{j} \alpha_{j}^{k}\left[Z_{j}^{k}\right]
$$


where $\alpha_{j}^{k}$ are nonnegative numbers that are independent of the metric. However the geometric meaning of these numbers is not clear to us.

\section{Some examples}

The first two examples suggest that not only the component $M_{p}$ of the current $M$ is of interest.

ExAmple 1. Let us assume that $X$ is compact, and that we have sections $f_{j}$ of rank $m_{j}$ bundles $E_{j} \rightarrow X$, such that $\sum m_{j}=n$. If $E=\oplus E_{j}$ and $f=$ $\left(f_{1}, \ldots, f_{r}\right)$, then the intersection number $v$ of the varieties $Z_{j}=\left\{f_{j}=0\right\}$ is equal to the integral of

$$
c_{n}(E)=c_{m_{1}}\left(E_{1}\right) \wedge \ldots \wedge c_{m_{r}}\left(E_{r}\right)
$$

over $X$. Since $M_{n}$ represents the cohomology class $c_{n}(E)$, we thus get the representation

$$
v=\int_{X} M_{n},
$$

i.e., an integral over the set-theoretic intersection $Z=\cap Z_{j}$. If $E$ is positive then $M_{n}$ is positive. If $Z$ is discrete, i.e., $f$ is a complete intersection, then $M_{n}=[Z]$, and in this case thus we just get the sum of the points in $Z$ counted with multiplicities, as expected.

ExAmPLe 2. Let $X$ be a compact Kähler manifold with metric form $\omega$, and let $f$ be a holomorphic section of $E \rightarrow X$. If moreover $E \geq_{B} 0$, then we know that $c\left(D_{E}\right), M$, and $c\left(D_{Q}\right) 0$ are all positive. Because of (1.4), we therefore have that

$$
\int_{X} M_{k} \wedge \omega_{n-k}=\int_{X} c_{k}\left(D_{E}\right) \wedge \omega_{n-k}-\int_{X} c_{k}\left(D_{Q}\right) \wedge \omega_{n-k} \leq \int_{X} c_{k}\left(D_{E}\right) \wedge \omega_{n-k} .
$$

Thus we get an upper bound of the total mass of $M_{k}$ in terms of the Chern class $c_{k}(E)$. Taking $k=p=\operatorname{codim} Z$ we get the estimate

$$
\operatorname{area}\left(Z^{p}\right)=\int_{X}\left[Z^{p}\right] \leq \int_{X} c_{p}(E) \wedge \omega_{n-p} .
$$

Example 3. Now assume that $X=\mathrm{P}^{n}$, let

$$
\omega=\aleph \partial \bar{\partial} \log |z|^{2}=d d^{c} \log |z|
$$

denote the Fubini-Study metric and notice that

$$
\int_{P^{n}} \omega^{n}=1,
$$


that is, the total area of $\mathrm{P}^{n}$ is $1 / n$ !.

Assume that $F_{1}, \ldots, F_{m}$ are polynomials in $C^{n}$ which form a complete intersection. If $F_{j}$ has degree $d_{j}$ (depending on $z^{\prime}=\left(z_{1}^{\prime}, \ldots, z_{n}^{\prime}\right)$ ) then the the homogenization $f_{j}(z)=z_{0}^{d_{j}} F\left(z^{\prime} / z_{0}\right)$ is a $d_{j}$-homogeneous polynomial in $\mathrm{C}^{n+1}$ and hence corresponds to a section of the line bundle $\mathcal{O}\left(d_{j}\right) \rightarrow \mathrm{P}^{n}$. Thus $f=\left(f_{1}, \ldots, f_{m}\right)$ is a section of $E=\oplus \mathscr{O}\left(d_{j}\right)$. If $E$ is equipped with the natural metric, i.e.,

$$
\|h([z])\|^{2}=\sum_{j} \frac{|h(z)|^{2}}{|z|^{2 d_{j}}}
$$

for a section $h=\oplus h_{j}$ of $E$ (here [z] denotes the point on $\mathrm{P}^{n}$ corresponding to the point $z \in \mathrm{C}^{n+1} \backslash\{0\}$ under the usual projection), then it is easy to check that $E \geq_{B} 0$. Therefore $M_{m} \geq 0$, and since moreover,

$$
\left.M_{m}\right|_{c^{n}}=[Z]
$$

if $Z$ here denotes the zero variety $\{F=0\}$ in $C^{n}$, then

$$
\operatorname{area}(Z)=\int_{C^{n}}[Z] \wedge \omega_{n-m} \leq \int_{\mathrm{P}^{n}} M_{m} \wedge \omega_{n-m}=\int_{\mathrm{P}^{n}} c_{m}\left(D_{E}\right) \wedge \omega_{n-m},
$$

since $c_{m}\left(D_{Q}\right)=0$. Here "area" refers to the projective area of course. However, $c\left(D_{E}\right)=\left(1+d_{1} \omega\right) \wedge \ldots \wedge\left(1+d_{m} \omega\right)$, and so

$$
c_{m}\left(D_{E}\right)=d_{1} \cdots d_{m} \omega^{m} .
$$

Hence

$$
\operatorname{area}(Z) \leq d_{1} \cdots d_{m} \frac{1}{(n-m) !} .
$$

We also notice that the deviation from equality is precisely the total mass of $M_{m}$ on the hyperplane at infinity. If $m=n$ we get Bezout's theorem

$$
\#\{F=0\} \leq d_{1} \cdots d_{n} .
$$

EXAMPLE 4. If $f$ is a complete intersection, i.e., $p=m$, and $W_{m-1}$ denotes the component of bidegree $(m-1, m-1)$, then

$$
d d^{c} W_{m-1}=c_{m}\left(D_{E}\right)-[Z]
$$

this means that $W_{m-1}$ is a Green current for the cycle $Z=\sum \alpha_{j} Z_{j}$.

In the case when $E=L_{1} \oplus \cdots \oplus L_{m}$ for some line bundles $L_{k}$, hence $c_{m}\left(D_{E}\right)=c_{1}\left(D_{L_{1}}\right) \wedge \ldots \wedge c_{m}\left(D_{L_{m}}\right)$, and $f=\left(f_{1}, \ldots, f_{m}\right)$, where $f_{j}$ are holomorphic sections of $L_{j}$, such a Green current was obtained already in [3]. 
ExAmPLE 5. Let $X$ be a compact manifold such that there is a holomorphic section $\eta$ of some vector bundle $H \rightarrow X \times X$ that defines the diagonal $\Delta \subset$ $X \times X$; for instance $X$ can be complex projective space. From Theorem 1.1 we get a current $W_{n}$ such that $d d^{c} W=c_{n}\left(D_{H}\right)-[\Delta]$. If we let $K(\zeta, z)=-W_{n}$ and $P(\zeta, z)=c_{n}\left(D_{H}\right)$, then

$$
d d^{c} K=[\Delta]-P,
$$

and this leads to the Koppelman type formula

$$
\begin{aligned}
\phi(z)-\int P(\zeta, z) & \wedge \phi(\zeta)=d d^{c} \int_{X} K \wedge \phi \\
& -d \int_{X} K \wedge d^{c} \phi+d^{c} \int_{X} K \wedge d \phi+\int_{X} K \wedge d d^{c} \phi
\end{aligned}
$$

for the $d d^{c}$-operator. In particular, if $\phi$ is closed $(k, k)$-form such that $d \phi=0$, then $d^{c} \phi=0$ as well, and thus

$$
v=\int_{X} K \wedge \phi
$$

is an explicit solution to $d d^{c} v=\phi-\int P \wedge \phi$. However if $X$ is non-compact one gets boundary integrals. It would be desirable to refine the construction to include somehow an appropriate line bundle with a metric that vanishes at the boundary, in order to obtain $d d^{c}$-formulas for, say, domains in $\mathrm{C}^{n}$.

EXAmple 6. Assume that $f$ is a holomorphic section of some Hermitian bundle $E \rightarrow X$ with zero variety $Z$. If $f$ is locally a complete intersection we have seen that the current $W_{m-1}$ from Theorem 1.1 is a Green current for [Z]. In general we have that $d d^{c} W_{p-1}=c_{p}\left(D_{E}\right)-c_{p}\left(D_{Q}\right)-\left[Z^{p}\right]$ so we only get a current $w$ such that $d d^{c} w=\left[Z^{p}\right]-\gamma$, where $\gamma$ is locally integrable. However, there is another and simpler way to find such a current $w$, due to Meo, [17].

Proposition 8.1 (Meo). Let $f$ be a holomorphic section of a Hermitian vector bundle $E \rightarrow X$. The forms

$$
w=\log |f|\left(\left(d d^{c} \log |f|\right)^{p-1} \mathbf{1}_{X \backslash Z}\right)
$$

and

$$
\gamma=-\left(d d^{c} \log |f|\right)^{p} \mathbf{1}_{X \backslash Z}
$$

are locally integrable on $X$ and

$$
d d^{c} w=\left[Z^{p}\right]-\gamma .
$$


For the reader's convenience we provide a simple proof based on Hironaka's theorem.

SKeTCH OF PROOF. Let $f=f_{0} f^{\prime}$ be as before, i.e., $f_{0}$ is holomorphic and $f^{\prime}$ is a non-vanishing section. Then $\log |f|=\log \left|f_{0}\right|+\log \left|f^{\prime}\right|$, and hence $d d^{c} \log \left|f^{\prime}\right|$ is smooth and $d d^{c} \log \left|f_{0}\right|=\left[f_{0}=0\right]$ has support on the inverse image $\tilde{Z}$ of $Z$ in the resolution. Thus

$$
w=\left(\log \left|f_{0}\right|+\log \left|f^{\prime}\right|\right)\left(d d^{c} \log \left|f^{\prime}\right|\right)^{p-1}, \quad \gamma=\left(d d^{c} \log \left|f^{\prime}\right|\right)^{p}
$$

are both locally integrable in the resolution and hence also on the original manifold. Moreover,

$$
d d^{c} w=\left[f_{0}=0\right] \wedge\left(d d^{c} \log \left|f^{\prime}\right|\right)^{p-1}+\gamma,
$$

in particular $\left(d d^{c} w\right) \mathbf{1}_{\tilde{Z}}$ is closed, and hence $T=\left(d d^{c} w\right) \mathbf{1}_{Z}$ is a closed current on $X$ of order zero. Therefore $T=\sum \alpha_{j}\left[Z_{j}^{p}\right]$, and since we can deforme the metric into a trivial metric locally, it follows from King's formula, [11], that $\alpha_{j}$ are precisely the multiplicities of $f$ on $Z_{j}^{p}$.

Assume now that $X$ is a compact manifold such that there exists a holomorphic section $\eta$ of some Hermitian bundle $H \rightarrow X \times X$ as in Example 5 above. If furthermore the kernel $K$ is reasonably regular we can assume that

$$
d d^{c} \int_{\zeta} K(\zeta, z) \wedge \psi(\zeta)=\psi(z)-\int_{\zeta} P(\zeta, z) \wedge \psi(\zeta)
$$

for any $(k, k)$-current $\psi$. We then have the explicit solution

$$
g=w+\int_{\zeta} K(\zeta, z) \wedge \gamma(\zeta)
$$

to the Green equation $d d^{c} g=\left[Z^{p}\right]-\alpha$, where $\alpha$ is the smooth form

$$
\alpha=\int_{\zeta} P(\zeta, z) \wedge \gamma(\zeta)
$$

The last example will be elaborated in a forthcoming paper.

\section{REFERENCES}

1. Andersson, M., Residue currents and ideals of holomorphic functions, Bull. Sci. Math. 128 (2004), 481-512.

2. Andersson, M., Residue currents of holomorphic sections and Lelong currents, Ark. Mat. 43 (2005), 201-219. 
3. Berenstein, C., and Yger, A., Green currents and analytic continuation, J. Anal. Math. 75 (1998), 1-50.

4. Berenstein, C. A., Gay, R., Vidras, A., and Yger, A., Residue Currents and Bezout Identities, Prog. Math. 114 (1993).

5. Berenstein, C., and Yger, A., Analytic residue theory in the non-complete intersection case, J. Reine Angew. Math. 527 (2000), 203-235.

6. Berndtsson, B., Cauchy-Leray forms and vector bundles, Ann. Sci. Éc. Norm. Sup. 24 (1991), 319-337.

7. Bismut J.-M., Gillet, H., and Soulé, Ch., Complex immersions and Arakelov geometry, The Grothendieck Festschrift, Vol. I, 249-331, Progr. Math. 86 (1990).

8. Bott, R., and Chern, S.-S., Hermitian vector bundles and the equidistribution of the zeroes of their holomorphic sections, Acta Math. 114 (1965), 71-112.

9. Chern, S.-S., Transgression in associated bundles, Internat. J. Math. 2 (1991), 383-393.

10. Demailly, J-P, Complex Analytic and Differential Geometry, Monograph Grenoble (1997)

11. King, J. R., A residue formula for complex subvarieties, Proc. Carolina conf. on holomoprhic mappings and minimal surfaces, Univ. of North Carolina, Chapel Hill (1970), 43-56.

12. Harvey, R., and Semmes, S., Zero divisors of atomic functions, Ann. of Math. 135 (1992), 567-600.

13. Harvey, R., and Lawson, B., A theory of characteristic currents associated with a singular connection, Astérisque No. 213 (1993), 268 pp.

14. Harvey, R., and Lawson, B., Geometric residue theorems, Amer. J. Math. 117 (1995), 829873.

15. Harvey, R., and Lawson, B., Singularities and Chern-Weil theory. II. Geometric atomicity, Duke Math. J. 119 (2003), 119-158.

16. Meo, M., Résidus dans le cas non nécessairment intersection complète, C. R. Acad. Sci. Paris Sér I Math. 333 (2001), 33-38.

17. Meo, M., Courants résidus et formule de King, Preprint (2003), Ark. Mat. 44 (2006), 149-165.

18. Passare, M., Tsikh, A., and Yger, A., Residue currents of the Bochner-Martinelli type, Publ. Mat. 44 (2000), 85-117.

DEPARTMENT OF MATHEMATICS

CHALMERS UNIVERSITY OF TECHNOLOGY AND THE UNIVERSITY OF GÖTEBORG

S-412 96 GÖTEBORG

SWEDEN

E-mail: matsa@math.chalmers.se 